\title{
ORIGEN COMUNAL E INSTITUCIONALIZACIÓN DE LA UNIVERSIDAD NACIONAL DEL CENTRO DEL PERÚ
}

\section{COMMUNITY ORIGIN AND INSTITUTIONALIZATION OF THE NATIONAL UNIVERSITY OF THE CENTER OF PERU}

\author{
Teódulo Gerardo Lázaro Aquino ${ }^{1 *}$ \\ tlazaro@uncp.edu.pe \\ ${ }^{1}$ Universidad Nacional del Centro del Perú, Junín, Perú
}

*Correspondencia: Teódulo Gerardo Lázaro Aquino. Email: tlazaro@uncp.edu.pe

Recibido: 11.08.21 | Aprobado: 29.08.21

\section{RESUMEN}

El artículo tiene por objetivo analizar la trayectoria de una organización universitaria desde su origen privado - comunal hasta su nacionalización, como desenlace de la acción colectiva, organización, participación, movilización y recursos de las comunidades campesinas, organizaciones sindicales, populares y económicas de la sierra central del Perú. Es decir, el origen e institucionalización de la Universidad Comunal del Perú (UCP) a Universidad Nacional del Centro del Perú (UNCP), que muestra etapas importantes como la creación de las comisiones organizadoras desde 1928, la configuración de las bases fundacionales, la inscripción en registros públicos, la oficialización de su funcionamiento el 16 de diciembre de 1959, la apertura de labores académicas y la nacionalización el 20 de diciembre de 1961, que marcaron su institucionalidad posterior. En lo académico propició la formación del capital humano regional; la incorporación y transferencia tecnológica hacia las comunidades y su entorno; así como la transformación social, económica y política de la región central, con una apuesta institucional ambiental. Palabras clave: Acción colectiva, comunidades campesinas, organización universitaria, institucionalización.

\begin{abstract}
The article aims to analyze the trajectory of a university organization from its private - communal origin to its nationalization, as a result of the collective action, organization, participation, mobilization and resources of the peasant communities, union, popular and economic organizations of the mountains. central Peru. That is, the origin and institutionalization of the Communal University of Peru (UCP) to the National University of the Center of Peru (UNCP), which shows important stages such as the creation of the organizing commissions since 1928, the configuration of the foundational bases, the enrollment in public records, the officialization of its operation on December 16, 1959, the opening of academic work and the nationalization on December 20, 1961, which marked its subsequent institutionality. Academically, it promoted the formation of regional human capital; the incorporation and transfer of technology to the communities and their environment; as well as the social, economic and political transformation of the central region, with an environmental institutional commitment.
\end{abstract}

Keywords: Collective action, peasant communities, university organization, institutionalization. 


\section{INTRODUCCIÓN}

No cabe duda, que la fundación de un centro superior de estudios universitarios fue una de las más caras aspiraciones de las poblaciones de la sierra central en el siglo XX, especialmente de Junín, Pasco, Huancavelica y Huánuco, teniendo como centro de gravedad a la ciudad de Huancayo, cuyo desenlace final se dio a fines de los años 50 con la fundación de la UCP.

Este acontecimiento tuvo lugar en el marco de un intenso proceso de organización y sindicalización del campesinado peruano: por un lado, el nuevo asalariado agrícola que se agrupó en la Federación de Trabajadores Azucareros y la Federación Nacional de Campesinos del Perú (FENCAP) y por otro, los campesinos arrendatarios, aparceros, junto a las comunidades campesinas en torno a la Confederación Campesina del Perú (CCP) (Sulmont, 1976). En Junín, la organización campesina estuvo ligada a la FENCAP de orientación aprista.

El liderazgo aprista se fue debilitando, por la convivencia del Partido Aprista Peruano (PAP) con Manuel Prado desde 1956, que significó su viraje a la derecha y motivó una aguda resistencia interna; que se agravó cuando el movimiento campesino entró en acción, que rebasó y fraccionó sus bases, perdió legitimidad en la conducción política de varias organizaciones sindicales. Entre 1960-1961, las movilizaciones campesinas del Valle de la Convención (Cusco) se extendieron al centro del país, cuyos conflictos fueron entre las comunidades y la Cerro de Pasco Corporation (CPC), las primeras reclamaban por tierras que habían sido tomadas de forma "tramposa" por dicha empresa (Zapata y Garfias, 2014).

En Junín a lo largo de los años 60 vivió un proceso de sindicalización y lucha de obreros textiles, minero-metalurgistas, maestros y campesinos, estudiantes universitarios y secundarios, cuya conducción era disputada por apristas, acciopopulistas, izquierdistas y democristianos. Por ejemplo, la Unión Sindical Departamental de Trabajadores de Junín (USDTJ), fundada el $1^{\circ}$ de mayo de 1945 , fue una de las protagonistas principales, estuvo afiliada a la Central de Trabajadores del Perú (CTP), creada el primero de mayo de 1944, en un primer momento bajo control del PCP, poco tiempo después, la dirección fue arrebatada por el PAP con el liderazgo de Luis Negreiros (Kapsoli, 1987).

Asimismo, en Junín hubo un peso muy fuerte de la perspectiva comunal, basada en la trascendencia de las comunidades tradicionales principalmente en el valle del Mantaro, que tenía el mayor número de comunidades en el país: 276 reconocidas oficialmente al 30 de junio de 1961, que agrupaban a 279,341 comuneros; seguido de Lima con 236 comunidades (Departamento de Junín tiene 276 comunidades, 1962).

Bajo el liderazgo de Elías Tácunan Cahuana se llegaron a constituir la Federación Provincial de Comunidades Campesinas de Huancayo (FCCPH) en diciembre de 1959 y la Federación de Comunidades Campesinas del Departamento de Junín (FCCDJ) en febrero de 1962, que jugaron un importante rol social, económico, político y cultural en la sociedad regional, de manera autónoma o articulando formas de organización y movilización junto a otros actores sociales y políticos, como evidencia del espíritu y acción colectiva regional, como el caso de la formación de la UCP. 


\section{UNIVERSIDAD: ORGANIZACIÓN E INSTITUCIÓN}

Las organizaciones y las instituciones son la base de la vida social, económica y política de la sociedad. Son reglas formales e informales, mecanismos de monitoreo y cumplimiento, sistemas de significados que definen el contexto dentro del cual individuos, corporaciones, sindicatos, naciones - estado y otras entidades operan e interactúan entre sí (Campbell, 2004).

Así, las universidades, en general, configuran y asumen diversas formas de organización e institucionalidad, en tanto formaciones sociales que aparecen en determinadas estructuras de oportunidades políticas. En este caso, los espacios de decisión que aparecieron al interior del Poder Legislativo y el Poder Ejecutivo, que fueron aprovechadas por la acción organizada de la sociedad civil que logró la creación de la organización universitaria.

Las estructuras organizacionales en general y las universidades en particular se crean y se hacen más complejas con el surgimiento de mitos. En contextos altamente institucionalizados, la acción organizacional se apoya en mitos, más allá de prestar atención a la actividad práctica para la que fue creada. La institucionalización de mitos racionales genera organizaciones más formales y complejas para la acción colectiva. Es decir, las organizaciones que incorporan mitos son más legítimas y exitosas, con mayores probabilidades de sobrevivir (Meyer y Rowan, 1991).

Cada organización universitaria estatal o privada identifica y consolida una identidad institucional a partir de mitos históricos, étnicos o comerciales, que busca construir un sentido de pertenencia interna (en la comunidad universitaria) y externa (en la sociedad regional, nacional e internacional). En el caso del estudio, el mito estuvo centrado en la identidad comunal, histórica y étnica, ya que adoptó el ticpe o ticpana huanca como escudo, sello y símbolo milenario, en tanto representación social de la comunidad campesina y la historia regional huanca.

Existen diversos enfoques para el análisis institucional como la teoría de la elección racional, que prioriza el microanálisis y asume que la acción está restringida por reglas como los derechos de propiedad, las constituciones y la racionalidad limitada. El institucionalismo organizacional que enfoca los ámbitos orgánicos y poblacionales y concibe la acción limitada por marcos culturales, esquemas y rutinas. Mientras para el institucionalismo histórico la acción está restringida por reglas y procedimientos, paradigmas cognitivos y creencias basadas en el macro análisis (Campbell, 2004).

El artículo asume las tres perspectivas, pues, las acciones de los involucrados en la creación de la UCCP tuvieron un enfoque de medio - fin, en el marco de una gestión nacional (gobierno central, poder legislativo), regional (participación de actores departamentales) y local (movilización de sujetos sociales y políticos desde y en la ciudad de Huancayo). La acción colectiva educacional tenía una aspiración concreta: la fundación de una universidad sea comunal o estatal, que confluyó a actores comunales, sociales, sindicales, empresariales y municipales, en el ámbito de la región central del Perú.

En el entendido que las instituciones son asentamientos nacidos de la lucha y la negociación, que reflejan el uso de recursos y el poder de quienes los crearon y, a su vez, afectan la distribución de los recursos y el poder en la sociedad (Campbell, 2004), se adoptan algunos conceptos de universidad como la siguiente: es un conjunto de personas 
que conforman una corporación; una institución dedicada a la enseñanza superior y a la investigación; y, un edificio o conjunto de edificios donde se imparte la enseñanza superior (Larousse, 2000).

Así, se parte del criterio que una universidad es una organización, que ha transitado por un proceso de formalización o institucionalización, traducido en estructuras orgánicas e institucionales más estables cuyos fines centrales son la investigación y la enseñanza superior, localizada en determinadas sedes universitarias que simbolizan su representación social. Y dado que la universidad es una forma social su institucionalización se caracteriza por presentar períodos de estabilidad y crisis, debido a factores internos como externos intervinientes en la vida organizacional.

Como señalan Magaña y Comas (2014), las universidades son entes ideados para producir conocimientos con edificaciones sociales muy complejas. Son organizaciones con procesos, relaciones, estructuras y cultura, donde confluyen personas y colectivos con objetivos comunes y particulares. Como instituciones (reglas, normas, políticas y regulaciones) impulsan la unidad y fomentan identidades. Así, en tanto organización institución se encuentra expuesta al entorno de forma permanente. Lo que sugiere aplicar y consolidar sistemas organizacionales de seguimiento y evaluación estratégica permanente.

Es decir, la universidad como institución social donde se funden aspectos sociales, culturales, raciales, históricos y sicosomáticos promueve y determina acuerdos sociales y, a la vez, organizan la vida de las personas vinculadas. El mismo origen de la universidad se asienta en imaginarios de poder, de dominio del conocimiento para ejercer influencias sobre las diversas esferas de la vida humana (Murcia y Gamboa, 2014). Sin embargo, la universidad, es más, presenta otra doble condición: es poseedora del capital cultural de la sociedad, a la vez, que promueve el capital cultural de los estudiantes.

Vale decir, la universidad apareció para producir conocimientos y mejorar los saberes en los distintos campos de la vida humana y la naturaleza. De allí la importancia de tenerla como objeto de estudio. Asimismo, la categoría de campo elaborada por Bourdieu permite contextualizar el análisis de la universidad en relación a otros sujetos, con sus propios recursos y estrategias, que les permiten la correlación de fuerzas a fin de lograr los fines organizacionales (Vargas, 2021).

\section{ORIGEN DE LAS UNIVERSIDADES NACIONALES O ESTATALES O PÚBLICAS}

Las universidades nacionales, estatales o públicas involucran a aquellas organizaciones educativas creadas o no por el estado, -siendo luego nacionalizadas-, cuyos presupuestos son financiados, casi en su totalidad, por el erario nacional, es decir, dependen económicamente del estado, aun cuando la norma sostenga su autonomía académica, económica y administrativa.

Ahora bien. El origen y fundación de las mismas tiene una historia singular, aun cuando comparten los mismos fines por ley, a diferencia de las universidades privadas (empresas de educación universitaria), cuyos objetivos son básicamente el lucro que, en el marco del neoliberalismo, han inundado el mercado educacional en su mayoría durante los últimos 25 años. 
Históricamente, la universidad como institución social surgió en el contexto social y cultural de la sociedad europea occidental urbana de los siglos XI y XII. Las circunstancias sociales favorables para su aparición fueron: a) el aumento de la población, que se tradujo en una permanente urbanización, b) los cambios en la organización social, como secuela de las transformaciones en las estructuras económicas, c) el surgimiento de una extraordinaria sed de saber d) la aparición de un nuevo oficio: la enseñanza como una de enfrentar las nuevas demandas requeridas por el proceso socio-cultural en curso (Tünnermann, 1992).

En el caso peruano, las primeras universidades públicas fueron: San Marcos (Lima) creada el 12 de mayo de 1551 y confirmada en 1571 como estatal y pontificia; San Cristóbal de Huamanga (Ayacucho) fundada en 1677 y ratificada dos veces (1680 y 1682); en 1692 se fundó la Universidad San Antonio Abad (Cusco). Todas relacionadas a órdenes religiosas que reprodujeron el modelo universitario hispano. En 1824 apareció la Universidad Nacional de Trujillo, la primera de la república, fundada por Simón Bolívar y José Faustino Sánchez Carrión, dando inicio al modelo universitario republicano. Prosiguió la Universidad Nacional San Agustín de Arequipa (1827) (Robles, 2006).

Como puede verse, las instituciones universitarias tuvieron distintos orígenes, con un punto en común: fueron creadas desde el poder dominante (Iglesia, Estado). El artículo se refiere a otra modalidad de formación, a una nueva forma social de educación superior, que surgió gracias a la capacidad de organización, movilización y propuesta de distintos entes de la sociedad civil. Es decir, fue el fruto de la acción colectiva de las organizaciones sociales al margen del poder dominante o más bien con el soporte de actores dominados, una forma de presión de los de abajo hacia los de arriba.

\section{ORIGEN DE LA UNIVERSIDAD COMUNAL DEL PERÚ (UCP) LAS COMISIONES ORGANIZADORAS Y SUS ACCIONES}

La historia regional de Junín registra dos momentos en la conformación de diversas comisiones organizadoras pro creación de una universidad en Huancayo. El primero, entre 1928 hasta 1958, cuyas gestiones estuvieron encaminadas junto a la representación parlamentaria, sin obtener resultados positivos.

Las comisiones organizadoras, que se renovaban cada cierto tiempo, tuvieron vida desde 1928, seguida del 25 de julio de 1942, del 16 de agosto de 1944. Entre 1947 y 1948, se enviaron oficios a diversas entidades, se organizó la asamblea general de febrero de 1948, con la presencia de representantes de organizaciones comunales dispuestas a cooperar en la organización y funcionamiento de la futura universidad. En agosto de 1950, se elevó otro memorial al Parlamento Nacional (Génesis y fundación de la Universidad de Huancayo, 1986 y Lahura, 1960a), que tampoco tuvo los resultados esperados.

La estrategia de incidencia combinó artículos de opinión, organización, movilización y gestión popular. El primero de marzo de 1958, a iniciativa de la Asociación de Estudiantes de Instrucción Superior de Huancayo (AEISH), en un congreso donde participaron comités departamentales, provinciales y distritales, delegados de diversas instituciones comunales, comerciales, industriales, bancarias, profesionales, culturales y laborales, se constituyó el Comité central pro universidad, que elaboró y presentó el Anteproyecto de Ley de creación y funcionamiento que, por antagonismos políticos del 
contexto, quedó encarpetado en la Comisión de la Cámara de Diputados (Solórzano, 1959 y Orihuela, 1959a).

El segundo momento decisivo en la historia de las comisiones organizadoras data

del 4 de marzo de 1959, cuando en asamblea convocada por la AEISH, se sustituyó la junta directiva del Comité central pro universidad, saliendo elegido Jesús Véliz Lizárraga como presidente, que encaminó dos estrategias: a) continuar con las gestiones ante el Congreso buscando la dación de la norma y b) incidir en comunidades, municipalidades y la población en general, para organizar una universidad municipal o comunal. Se apostó por la segunda, por una universidad particular, antes de seguir esperando la buena voluntad del Estado.

Cabe mencionar que otra forma de presionar la creación de la nueva universidad, consistió en la conformación de comités locales pro universidad, como filiales del comité central, en cada lugar de trabajo (anexo, distrito o capital de provincia) de los maestros acreditados de los niveles de primaria y secundaria en los departamentos de Pasco, Huancavelica, Huánuco y Junín. Otras fueron constituidas por las propias municipalidades.

Así, varios comités distritales pro universidad, como muestra de apoyo, convocaron a concentraciones masivas como en Chongos Alto, Huasicancha y Carhuacallanga en la zona alta de la provincia de Huancayo, con la asistencia de delegaciones de los distritos de Colca, Jarpa y Chacapampa; mientras a San Jerónimo de Tunán (Huancayo) concurrieron delegaciones de los distritos de San Agustín de Cajas, Hualhuas, San Pedro de Saño, Quilcas, Ingenio y Quichuay. Por su parte, los comuneros de San José de Quero (Concepción), en asamblea pública acordaron aportar con tres mil soles para la gestión.

Sin embargo, se debe resaltar la ausencia de algunas comunidades organizadas del Valle del Mantaro como Muquiyayuyo, Huaripampa, Muqui, Sincos, Masma, Julcán, Huamalí, Mantaro, que constituían las fuerzas más poderosas en lo comunal y de varios concejos distritales (Orihuela, 1959b).

En ese contexto, el Comité central pro universidad encargó la culminación de la elaboración del proyecto a los Catedráticos Javier Pulgar Vidal, como asesor principal, Jorge Chiriboga, Luis Rodríguez y Carlos Enrique Ferreyros (vicepresidente de la Cámara de Diputados) en coordinación con las organizaciones comunales, municipales y populares. Las líneas iniciales eran la creación de una universidad comunal, no un remedo de las universidades tradicionales, que abarcara nuevos aspectos de la enseñanza. Una universidad que represente el aporte de las comunidades de la región central.

\section{LA CONCEPCIÓN INICIAL DE LA NUEVA UNIVERSIDAD}

El 10 de mayo de 1959, en asamblea general, con la asistencia de más de 60 delegaciones de comunidades, municipios, sindicatos y otras entidades de la región central, se fijaron las bases para la creación de la UCP, en los aspectos académicos, técnicos y administrativos. Para entonces ya había la decisión política de crear una universidad comunal, aunque el tema financiero seguía siendo el problema principal.

Sobre la concepción de la nueva universidad, Véliz (1959) presidente de la Comisión inicialmente delineó lo siguiente: La base filosófica se centraría en el neohumanismo, el 
pragmatismo y el indoamericanismo (por su filiación aprista). El neo humanismo buscaría el perfeccionamiento del hombre tanto en lo profesional como en lo técnico, buscando la calidad humana, donde el hombre sea el centro, su dignidad y libertad. Una preparación profesional al servicio de la sociedad. El pragmatismo daría al universitario una especialización técnica, el sentido práctico de la formación del hombre-técnico, como una parte del perfeccionamiento humano, complementado al neohumanismo.

La universidad debería formar al hombre que ponga la técnica a su servicio y no al hombre supeditado a la técnica. El indoamericanismo, sin ser una corriente filosófica, serviría para ubicar al hombre dentro de su realidad, debiendo partir la solución de los problemas de la propia realidad en que se vive. En suma, neo humanismo, pragmatismo e indoamericanismo se complementarán para formar al hombre técnico y humanista al servicio de la región y del país, lo que constituye su verdadera misión.

La base académica se basaría en las necesidades de la región. Se propuso las facultades de Agronomía, Veterinaria, Medicina, Ciencias Sociales y Políticas, Ingeniería, Ciencias Económicas y Comerciales, Educación y Recursos Naturales. Otro aspecto académico importante serían los Institutos de Investigación Científica, que coordinarán sus actividades con las Facultades. Estas serían de Matemática, Ciencias Biológicas, Ciencias Fisicoquímicas, Investigaciones Sociales y Económicas y Educación. Las acciones servirían para la preparación de profesionales y consultores para diferentes organismos estatales y privados, estar en contacto con la realidad regional y nacional.

En relación a las bases económicas, la financiación podría hacerse bajo dos formas: si fuera creada por ley como Universidad Nacional, el Estado sería el único encargado de la misma. De lo contrario, sería una universidad auspiciada por los municipios y las comunidades de la región, para lo cual se requería de un plan técnico de financiación.

La base administrativa de la Universidad sería un patronato integrado por delegados de comunidades, municipalidades, empresas, accionistas particulares, la propia universidad y los estudiantes, quienes formarían un Consejo de Administración que causaría la marcha económica y administrativa. En lo académico, tendría un Consejo Universitario integrado por el rector, decanos, directores de Institutos y delegados de los catedráticos. Ambos consejos estarían en constante coordinación.

En consecuencia, la Universidad Comunal tendría las siguientes características: 1) Docencia organizada científicamente; 2) Creación de un clima propicio para la investigación; 3) Estudio de los problemas regionales y nacionales; 4) Promoción de la cultura; 5) Desarrollo de programas sistemáticos de extensión cultural y servicio social; 6) Ser exponente de los más altos valores regionales y nacionales, un centro promotor y mantenedor de los más elevados ideales humanos en el seno de la sociedad. 7) Mantener vivo en la juventud los sentimientos profundos y equilibrados de nacionalidad, constante superación y deseo de saber para servir.

Asimismo, la universidad comunal era concebida como uno de los principales actores para el desarrollo regional y del valle del Mantaro, siendo uno de sus objetivos la preparación de profesionales para el desarrollo planificado del país (Solís, 1960). 


\section{CONSTITUCIÓN Y FORMALIZACIÓN DE LA UNIVERSIDAD COMUNAL}

La nueva universidad se constituyó ante notario público como privado. Respecto a la Escritura Pública de Constitución existen dos versiones, según la primera, fue celebrada el 11 de julio de 1959, en la Notaría Pública de Marino Lahura Olivo, con el nombre de Universidad Comunal del Perú (UCP); la segunda del 15 de septiembre de 1959, mencionada en el Decreto Supremo No 59, que oficializó su funcionamiento.

Al respecto, señala Orihuela (1959b), el Comité pro universidad con el soporte de más de 100 sub-comités en los departamentos de Huancavelica, Junín, Pasco y Huánuco, elaboró y presentó un anteproyecto ante la Cámara de Diputados como primer intento y ante la eventualidad de un fracaso se tuvo la opción de crear una entidad de derecho privado. Véliz siguió la segunda alternativa fundando una asociación civil denominada universidad comunal" sin fines de lucro" de conformidad al art. 44 del Código Civil.

Los miembros del primer Consejo Directivo de la UCP fueron: Javier Pulgar Vidal (Rector), Jesús Véliz Lizárraga (director gerente), Víctor Alfaro de la Peña (director financiero), José Illanes Torino (director de coordinación), Wilfredo Paredes (director de economía), Luis Felipe Caballero (director de relaciones públicas) y Luis Vega Fernández (director de entidades sostenedoras). Como se observa la estructura orgánica era propia de una entidad privada.

Al respecto, el capítulo segundo de la Ley universitaria 10555, reconocía que la universidad se gobernaba a través del Consejo Universitario, los Consejos de Facultad y la Junta Directiva de las Escuelas e Institutos y tenía como autoridades al rector, vicerrector, decanos, subdecanos, directores y sub directores. Mientras la Ley 13417 diferenciaba dos tipos de personas jurídicas: las universidades estatales de derecho público interno y las universidades particulares de derecho privado; sobre los órganos de gobierno consideraba al rectorado, consejo universitario y asamblea universitaria; y, como autoridades al rector, vicerrector y decanos de facultad.

Finalmente, el 16 de diciembre de 1959, se oficializó el funcionamiento de la universidad comunal mediante el Decreto Supremo 46, firmado por Manuel Prado, presidente de la República. La formalización fue consecuencia de la presión popular. En el documento se destacaba a los fundadores: comunidades de indígenas, concejos municipales, asociaciones, clubes, sindicatos, centros sociales, empresas comerciales e industriales, colegios particulares y personas, que por el sistema de ayuda mutua hicieron posible la acumulación de bienes inmuebles y capital.

Asimismo, se hacía mención a la Escritura Pública de Constitución $\mathrm{N}^{\circ} 371$, bienio 1959-1960, suscrita el 15 de septiembre de 1959, al Estatuto, a los fundadores y asociaciones de la Universidad Comunal que se comprometían a sostener de manera indefinida la vida institucional. También se reconocía la fundación y se autorizaba el funcionamiento de la UCCP, con carácter particular con sede en la ciudad de Huancayo. Se aprobaba el estatuto, el reglamento general y el plan de estudios con las modificaciones sugeridas. Se facultó al consejo directivo la convocatoria a plazas de decanos y profesores, a exámenes de selección de estudiantes.

Así como se reconocerían los certificados de estudios y los grados académicos que emitiera hacia adelante. Siendo creada con doce facultades: Economía, estadística y finanzas; Ciencias sociales y seguridad social; Administración pública y privada; 
Contabilidad, comercio y cálculo actuarial; Educación comunal; Ciencias geográficas y planeamiento; Recursos naturales y Ciencias forestales; Zootecnia; Acuicultura y oceanografía; Arquitectura social e Ingeniería industrial.

Por ello, Loyola (1960) resaltó que la universidad comunal nació por las necesidades regionales, nacionales e individuales. Además, de enfatizar que pocas comunidades lograron organizar una universidad, por lo que este acontecimiento era un motivo suficiente para que las comunidades, instituciones, municipalidades, organizaciones sindicales, profesionales y culturales sean acreedoras de la gratitud de las futuras generaciones.

Sin embargo, no está clara en el marco de qué ley universitaria se creó y formalizó la UCCP, puesto que la Ley 10555 de 1946 -dada por Bustamante y Rivero- fue derogada después del golpe de estado de 1948, protagonizada por Odría, y recién en abril de 1960 Prado promulgó la Ley 13417. La institucionalización sí se hizo bajo la última norma.

\section{INSTITUCIONALIZACIÓN: DE UNIVERSIDAD COMUNAL A UNIVERSIDAD NACIONAL}

Como pudo verse, la UCCP fue creada y formalizada como ente privado, quedando hacia adelante el reto de consolidar su institucionalización académica, administrativa, económica - financiera y social, con el compromiso asumido por las entidades fundadoras y el consejo directivo. Para el cual, se implementaron diversas estrategias y rutinas como parte de la construcción institucional, que terminó con la nacionalización o estatización, donde empezó otra etapa que perdura hasta hoy.

\section{INAUGURACIÓN INSTITUCIONAL DE LA UCCP}

El acto de inauguración institucional y de la sede de la UCCP fue el 20 de diciembre de 1959, de forma apoteósica. Hubo doce madrinas para igual número de facultades. Estuvieron presentes las comunidades tradicionales, los sindicatos, los concejos municipales, las empresas, las asociaciones, los clubes, las delegaciones universitarias, las escuelas especiales y los colegios, y otras personas provenientes del campo y otras ciudades, concentrados por el gran día inicial de la universidad comunal (Lahura, 1960b).

\section{APERTURA DE LAS LABORES ACADÉMICAS DE LA UCCP}

El 3 de abril de 1960, marcó otro hito en la historia regional. Los estudiantes de la universidad comunal, rodeados de padrinos, Embajadores de 12 naciones y acompañados por estudiantes, funcionarios, empleados, obreros y comunidades campesinas, desfilaron por primera vez por la histórica Calle Real (Navarro, 1960) de la ciudad de Huancayo.

Además, el acto oficial de inauguración de las labores académicas fue el mismo día a las 7 p.m. con la presencia de Luis Alvarado, ministro de Trabajo y Asuntos Indígenas. Para José Rubio, ministro de Educación Pública, la apertura del año académico constituía un acontecimiento en la vida cultural de los departamentos de la región central y del país, ya que contribuiría al progreso de los conocimientos técnicos, académicos y humanísticos. Asimismo, resaltó la acción y cooperación de la comunidad que, amparada por el gobierno, hizo posible la fundación de la nueva universidad. 
La implementación de los ciclos académicos mostró el crecimiento y consolidación de la universidad comunal. En la sede central de Huancayo funcionaban las doce facultades, en Lima tres, en Huacho una, mientras se fundaba otra filial en Cerro de Pasco.

De forma paralela, la universidad se involucró en la realidad regional, organizando disertaciones públicas; a través de la Facultad de Ciencias Sociales participó en la Escuela Sindical del Centro, orientada a los gremios sindicales de los departamentos de Junín, Pasco, Huánuco, Huancavelica y Ayacucho. Asimismo, auspició el funcionamiento de escuelas y colegios comunales de nivel secundario en Huancayo, San Jerónimo de Tunán, Chongos Alto y Manzanares (Concepción).

\section{PRIMERA CRISIS INSTITUCIONAL DE LA UCCP}

El primer año de funcionamiento fue una verdadera prueba de fuego para su institucionalización. Así, a fines de 1960, circuló la versión de que la universidad dejaría de funcionar en 1961. En ese momento, tenía facultades en Huancayo, Lima, Huacho, Cerro de Pasco y Huánuco, con aproximadamente dos mil alumnos. Siendo el aspecto económico - financiero el escollo principal.

Durante la gestación de la universidad, el Comité Central había recaudado aproximadamente 80 mil soles, el mayor porcentaje provino de donativos y la organización de diversos eventos. Para el funcionamiento llegaron los aportes de mil soles del Colegio Particular Santa Teresita, 15 mil de los fabricantes de Cerveza "Cristal", 10 mil de la Compañía Nacional de Cerveza Callao, 11 mil del Comité Distrital de Huayllay, 5 mil del Sindicato de Choferes, 5 mil del Sindicato Textil de Manufacturas del Centro, entre otros. Por su parte, el Senado de la República aprobó la consignación de 700 mil soles. De hecho, insuficientes para la sostenibilidad de la novel entidad.

Ante la preocupación del estudiantado universitario, en enero de 1961, se conformó el Comité provisional pro nacionalización de la UCCP, a fin de asegurar su estabilidad y permanencia, ante la oposición de las organizaciones comuneras. Para cuyos dirigentes el comité pretendía destruir a la universidad, fundada para el progreso de las comunidades tradicionales, siendo tildado de politiquero y traidor a la causa de las comunidades y del pueblo peruano. Además, exhortaron a su "no estatalización", con el pretexto de “nacionalización”. Entre los firmantes estuvo Elías Tácunan Cahuana, uno de los gestores principales de la universidad comunal.

El impulso de la "nacionalización" de la universidad privada - comunal había quebrado las relaciones con las comunidades, que tanto había apoyado para su fundación. Sin embargo, a pesar de la oposición de los dirigentes campesinos, el comité provisional también por iniciativa de la AEIHS, convocó a otra asamblea para elegir al Comité central permanente pro nacionalización. Los catedráticos de la UCCP manifestaron su conformidad con la formación de dicho comité y, a la vez, conformaron una Junta Reorganizadora Provisional con el apoyo de las facultades.

Frente a la grave crisis interna, por acuerdo de la Junta Reorganizadora encabezada por Augusto Peñaloza Vizcarra y la UCCP representada por Javier Pulgar Vidal, se constituyó un consejo académico y un consejo administrativo, con la finalidad de asegurar su funcionamiento normal. 
En medio de la crisis y los vientos de nacionalización, la universidad celebró el primer aniversario y conmemoró el funcionamiento de las doce facultades, coincidiendo con el inicio del Tercer Ciclo Lectivo Académico. Además, se colocó la primera piedra de la "Ciudad Universitaria", ubicada en el Barrio de "Yauris" en el distrito de El Tambo.

En ese contexto, recordó Vásquez (1961), que la UCCP era el alma de la comunidad tradicional peruana, porque surgió del espíritu comunal como máxima expresión de cultura, cuya filosofía, principios y fines la distinguían de otras universidades del país. Por lo tanto, la educación que imparte debía basarse en la investigación científica de la realidad socio-económica, cultural y educativa, que consolide una cultura peruana basada en el pensamiento comunal; una educación que sea expresión del pueblo peruano y sustento de la democracia plena de Libertad y valores.

Dado que la nacionalización de la UCCP había generado expectativa, se realizó un mitin en solidaridad con la Federación de Estudiantes de la Universidad Comunal del Centro del Perú (FEUCCP), que se centró en pedir la nacionalización y condenar los atropellos en contra de sus dirigentes. Asistieron Max Hernández presidente de la Federación de Estudiantes del Perú (FEP) y otros dirigentes estudiantiles de la Universidad Nacional Mayor de San Marcos (UNMSM) y de la Escuela Normal "Enrique Guzmán y Valle".

Mientras tanto, hubo acusaciones públicas de actos inmorales e irregularidades en el manejo de los recursos financieros y económicos de la UCCP, cuyo Rector era Javier Pulgar Vidal. Quien de héroe pasó a ser villano, por el contexto de conflicto institucional. Por su lado, las facultades de Contabilidad, Economía, Arquitectura y Ciencias Forestales, el 26 de mayo de 1961, iniciaron una huelga indefinida, la primera desde su creación, debido a que las autoridades impidieron la matrícula de siete estudiantes, por el hecho de haber participado e impulsado su nacionalización.

Hubo otro mitin convocado por el Comité pro nacionalización, donde se sostuvo el derecho a tener una universidad nacional financiada por el estado. Participaron los dirigentes sindicales José Suárez, Constantino Herrera, Oswaldo Peñaloza, Javier Ríos Burga, Antonio Cisneros, Saúl Muñoz, entre otros.

Asimismo, durante un conversatorio realizado en la universidad, delegados de nueve facultades y los catedráticos apoyaron la reorganización total. Se conformó el Frente Universitario de Reorganización, con el objetivo de alcanzar la reestructuración desde la Escritura de Constitución, Estatutos y Reglamentos. Se partía de la idea que la reestructuración era el primer paso hacia la nacionalización de la UCCP.

Frente a los problemas internos y la posición asumida por las facultades, para el Rector Javier Pulgar Vidal, la intervención del gobierno era imposible en un régimen de derecho, ya que era azuzado por el denominado Frente Universitario de Reorganización, un grupo sedicioso integrado por personas de conocida filiación política. En el mismo sentido, para los directivos de la UCCP, la huelga iniciada por los profesores no tenía fundamento jurídico, académico ni administrativo.

\section{PRIMERA COMISIÓN INVESTIGADORA DE LA UCCP}

En este escenario, como corolario de la petición de las organizaciones populares, sindicales, catedráticos y estudiantes, el Gobierno Central nombró una Comisión 
Investigadora, el 21 de julio de 1961, para que emitiera un informe sobre la situación de la UCCP y sus filiales, en un plazo de 45 días. Además, de sugerir medidas para su mejor organización, sin desvirtuar el espíritu de su creación y asegurar la no interrupción de su funcionamiento. La Comisión estuvo integrada por Roberto Kock (presidente), José Illanes Torino, Horacio Gutiérrez Tordoya, Virgilio Mora Alvis y Carlos Alberto Vásquez.

Al respecto, el Comité Universitario de Huelga a través del estudiantado expresó su posición de alerta y vigilancia en torno a las decisiones que tomará la Junta Investigadora, ya que no se trataba sólo del cambio de personas, sino de una transformación integral. Para el Comité pro nacionalización, la estabilidad y la conversión era una necesidad urgente y un ideal de años, pues la UCCP era concebida como un bien público y no particular.

Una vez que la Comisión Investigadora asumió la administración de la universidad, nombró dos Consejos: el Académico conformado por decanos; el Administrativo integrado por la Comisión Investigadora. Cabe señalar que en las universidades los órganos de gobierno se denominan "Consejos", mientras en las municipalidades se llaman "Consejos".

Por su parte, el Consejo Directivo de la UCCP acordó enjuiciar a la Comisión Investigadora por la supuesta violación de la estructura jurídica de la universidad, insinuando que no tenía facultades para ello y por haber transgredido otras disposiciones legales. En ese contexto, Elías Tácunan Cahuana director de Entidades Sostenedoras y secretario general de la Federación de Comunidades de la provincia de Huancayo (FCPH), fue nombrado como Personero Jurídico y asumió la defensa jurídica de la universidad.

La FCPH rechazó los acuerdos del Consejo Académico, por considerarlos una violación a las normas jurídicas de la universidad, censuró a la Comisión Investigadora y siguió defendiendo la filosofía, la organización y la estructura de origen de la UCCP, además de solicitar su participación en la dirección de la universidad, fundado en que la contribución económica les otorgaba el derecho de propiedad sobre este bien cultural.

En ese marco, el 20 de octubre de 1961, la Comisión Investigadora culminó su Informe, en el que recomendaba la nacionalización (estatización) como único medio posible para que cumpla con su verdadera función. Era el fin de la etapa inicial de la UCCP como entidad privada para pasar a formar parte de la institucionalidad de la educación superior universitaria estatal.

\section{NACIONALIZACIÓN DE LA UCCP}

En noviembre de 1961, el Diputado por Junín Alfredo Sarmiento Espejo presentó un proyecto de Ley que consideraba a la UCCP de carácter nacional en el marco de la Ley No 13417. Se basó en tres razones: los sucesos públicos y notorios producidos en torno al funcionamiento de la universidad, que crearon un clima de malestar y descontento entre catedráticos, alumnos y ciudadanos; el Congreso Nacional Extraordinario de la FEP que acordó solicitar su nacionalización; y, la Comisión Investigadora del Ministerio de Educación Pública, había manifestado por lo mismo.

Por su parte, los otros diputados por Junín, Eduardo Vallejos y Max Espinoza, también presentaron conjuntamente un proyecto de Ley de nacionalización. Ante ello, la FPCH los declaró traidores a la causa de las comunidades. Se supo, además, que en marzo 
la Federación junto al Consejo Directivo de la UCCP, habían solicitado el cambio de comunal a nacional, similar a la Universidad Católica. Se oponían a su estatización con el argumento de que si el Estado la nacionalizaba se estaba en contra de la obra creada por las Comunidades Tradicionales (Diputados por nacionalización de UC, 1961).

El 10 de noviembre de 1961, un promedio de 600 alumnos de la sede de Huancayo y la filial de Lima, se movilizaron en La Capital encabezados por dirigentes de la FEUCCP, la FEP, la UNMSM, la UNI (Universidad Nacional de Ingeniería) y la PUCP. Una Comisión estudiantil conformada por Fernando Romero, presidente de la FEUCCP y Max Hernández, presidente de la FEP, fue recibida por el presidente de la Cámara de Diputados. A la misma hora una Comisión de Catedráticos, acompañados por Roberto Koch, presidente de la Comisión Investigadora se entrevistó con el ministro de Educación. En ambos encuentros se les aseguró la pronta nacionalización de la UCCP.

Por su parte, la Cámara de Diputados, por abrumadora mayoría, aprobó la dispensa del trámite de comisión del proyecto de Ley presentado por Eduardo Vallejos Montenegro y Max Espinoza Galarza, para nacionalizar la universidad. La propuesta contemplaba que una Comisión nombrada por el Ejecutivo estudiará la adaptación de la UCCP a la Ley Universitaria en un plazo de tres años. El proyecto presentado por Sarmiento pedía su inmediata adaptación a la Ley Universitaria No 13417.

No obstante, continuaron las manifestaciones públicas para la nacionalización de la universidad, especialmente de los estudiantes universitarios, que solicitaron el apoyo de la clase trabajadora para iniciar una huelga indefinida conjunta. Incluso se levantó una barricada frente al local central en la Calle Real y la Capilla de La Merced en el centro de la ciudad de Huancayo.

El 20 de diciembre de 1961, la Cámara de Senadores aprobó la Ley N 13827, que nacionalizó a la UCCP como "Universidad Nacional del Centro del Perú" (UNCP), con sede en la ciudad de Huancayo, junto a las filiales de Lima, Cerro de Pasco, Huánuco y Huacho.

A pesar de los desencuentros y divergencias que hubo con los dirigentes de las comunidades campesinas, especialmente con la FPCH liderada por Elías Tácunan, durante el contexto de nacionalización de la UCCP, De la Vega (1961) remarcó que por primera vez se vio la participación de las comunidades indígenas en una obra de gran trascendencia histórica como fue la gestación de la universidad comunal y su nacionalización por el Congreso de la República. Además, de resaltar la importancia de las comunidades de Junín, que hicieron posible la creación de la organización universitaria.

El 20 de enero de 1962, a través de la Resolución Suprema Nº13, se formó la Comisión Organizadora de la UNCP designándose tres delegados del Ministerio de Educación. Según disponía el art. $3^{\circ}$ de la Ley $N^{\circ}$ 13827, dicha comisión debía estar integrada, además, por dos delegados elegidos por los catedráticos y dos delegados de la FEUNCP, bajo la Presidencia de uno de los delegados del Ministerio mencionado.

La Comisión Organizadora quedó conformada de la siguiente manera: Ing. Jorge Succar Rahme (UNI), Dr. Oscar Herrera Márquez y Dr. Manuel Vegas Castillo (UNMSM), como representantes del Ministerio de Educación. Los delegados elegidos en la UNCP fueron, por los catedráticos: Ing. Manuel Carranza Márquez y Dr. Joaquín 
Chivilchez Chávez y por los estudiantes: Fernando Romero Villanes y Julio Seminario Wengel.

El 7 de julio de 1962, en sesión de Consejo Universitario, el Dr. José Ignacio Vigil, representante del Ministerio de Educación Pública, hizo entrega del gobierno de la UNCP a las nuevas autoridades, poniendo fin a la grave crisis institucional. Tras la normalización del funcionamiento de la universidad, correspondía al personal docente, administrativo y alumnado, la responsabilidad de ganar el prestigio resquebrajado. Previamente, el Ing. Manuel Carranza Márquez había sido elegido en Asamblea Universitaria como primer Rector de la UNCP.

Así culminó una etapa y empezó una nueva travesía para la novel organización universitaria que costó mucho esfuerzo a la colectividad juninense y la región central del Perú.

\section{CONCLUSIÓN}

El origen, funcionamiento e institucionalización de la Universidad Comunal luego Nacional, entre 1959 y 1962, fue una cadena de acontecimientos organizativos, normativos y conflictivos, la movilización de todo tipo de recursos, por parte de las organizaciones comunales, sindicales, populares, municipales y empresariales de la región central. Podría decirse que se transitó de una institucionalidad social hacia una institucionalidad autónoma, propia.

La institucionalización en su primera etapa no fue nada fácil, continúa siendo compleja hasta hoy. Pero la creación de la universidad comunal y luego nacional tuvo un enorme impacto en la vida política, social, económica y cultural de la región central y del país.

Tres procesos de institucionalización marcaron la historia de la universidad: El primero, propiamente de organización inicial, a partir del estatuto proveniente de la escritura pública notarial, que duró hasta el 16 de diciembre de 1959, cuando se formalizó como Universidad Comunal; sin actividades académicas, sino de movilización social.

El segundo, empieza cuando inicia sus actividades de organización, cuya tarea más inmediata fue la convocatoria a examen de ingreso, tanto para estudiantes como docentes. Las actividades académicas empezaron con escasos recursos financieros, provenientes básicamente de las organizaciones de la sociedad civil, que financian las acciones académicas y administrativas. Es un período de crisis generalizada que termina en su nacionalización o estatización, el 20 de diciembre de 1961. Al año de su creación.

El tercero, no exento de dificultades empezó el julio de 1962, cuando se restablecieron sus labores académicas y administrativas, con nuevas autoridades, el estatus de universidad nacional y su adecuación a la normatividad en curso.

\section{ORCID}

Teódulo Gerardo Lázaro Aquino: Universidad Nacional del Centro del Perú, Junín, Perú

\section{FUENTE DE FINANCIAMIENTO}

El estudio fue autofinanciado. 


\section{CONFLICTOS DE INTERÉS}

El autor declara que no existe conflicto de intereses.

\section{AGRADECIMIENTO}

No aplica.

\section{PROCESO DE REVISIÓN}

Este estudio ha sido revisado por pares externos en modalidad de doble ciego.

\section{DECLARACIÓN DE DISPONIBILIDAD DE DATOS:}

No aplica.

\section{REFERENCIAS BIBLIOGRÁFICAS:}

Campbell, J. (2004). Institutional change and globalization. Princeton University Press.

De la Vega, R. (29 de diciembre de 1961). La Universidad Nacional del Centro. La Voz de Huancayo, p. 2.

Diputados por nacionalización de UC. (6 de noviembre de 1961). La Voz de Huancayo, p. 3.

Génesis y fundación de la Universidad de Huancayo. (15 de diciembre de 1986). Diario Correo de Huancayo, p. 8.

Kapsoli, W. (1987). Los movimientos campesinos en el Perú. Ediciones Atusparia. URL: https://es.scribd.com/document/324585691/Los-Movimientos-Campesinos-enEl-Peru

Lahura, M. (7 de enero de 1960a). Universidad y Comunidades del Valle. La Voz de Huancayo, p. 3.

Lahura, M. (23 de mayo de 1960b). Universidad transcendente. La Voz de Huancayo, p. 2.

Larousse (2000). Gran diccionario de la lengua española. Tomo 12. SPES Editorial.

Departamento de Junín tiene 276 comunidades. (9 de enero de 1962). La Voz de Huancayo, p. 3.

Loyola, C. (8 de abril de 1960). La Universidad Comunal del Centro. La Voz de Huancayo, p. 2 .

Magaña, L. y Comas, O. (2014). La universidad: ¿institución organizada, organización institucionalizada, o estatus híbrido? En R. Ceballos y L. Hernández (Comp.) XII Congreso Internacional de Análisis Organizacional Organizaciones e instituciones: tradición, desarrollo y multiculturalidad. Universidad del Caribe, Universidad de Quintana Roo y Red Mexicana de Investigadores en Estudios Organizacionales. Pp. 58-70. URL: https://www.researchgate.net/publication/283081753_La_Univer sidad_Institucion_organizada_organizacion_institucionalizada_o_estatus_hibrido /link/5629284108ae518e347c9562/download

Meyer, J. \& Rowan, B. (1991). Institutionalized organizations: Formal structure as myth and ceremony. En: W. Powell and P. Di Maggio (Edit). The new institutionalism in organizational analysis. The University of Chicago Press. 
Murcia, N. y Gamboa, A. (2014). Saber, manipulación y poder. La universidad como institución social. Hallazgos, número 22, 419-434. URL: https://www.researchgate.net/publication/273917076_Saber_manipulacion_y_p oder_La_universidad_como_institucion_social

Navarro, G. (8 de abril de 1960). Adelante estudiantes de la Universidad Comunal. La Voz de Huancayo, p. 2.

Orihuela, B. (4 de marzo de 1959a). Algunas labores del Comité Pro Universidad Nacional de Junín. La Voz de Huancayo, p. 5.

Orihuela, B. (19 de diciembre de 1959b). Algo sobre la Universidad Comunal. La Voz de Huancayo, p. 2.

Robles, E. (2006). Origen de las Universidades más antiguas del Perú. Revista Historia de la Educación Latinoamericana, volumen 8, 35-48. URL: https://www.redalyc. $\mathrm{org} / \mathrm{pdf} / 869 / 86900803 . \mathrm{pdf}$

Solís, C. (19 de mayo de 1960). La Universidad Comunal y el Desarrollo futuro de la Región del Valle del Mantaro. La Voz de Huancayo, p. 2.

Solórzano, R. (1 de marzo de 1959). ¡Por una Universidad de Huancayo! La Voz de Huancayo, P. 3.

Sulmont, D. (1976). El Movimiento Sindical en un Contexto de Reformas: Perú 19681976. Nueva Sociedad, 26, 39-62. URL: https://nuso.org/articulo/elmovimiento-sindical-en-un-contexto-de-reformas-peru-1968-1976/

Tünnermann, C. (1992). La Universidad: historia y reforma. Editorial UCA. URL: https://www.enriquebolanos.org/media/publicacion/3159.pdf

Vargas, G. (2021). Aproximación a los conceptos de campo, habitus, capital y violencia simbólica de Bourdieu. Revista Puriq, 3, 2, 327-344. DOI: https://doi.org/10.37073/puriq.3.2.166

Vásquez, Z. (5 de abril de 1961). La Universidad Comunal es el alma de la Comunidad. La Voz de Huancayo, p. 2.

Véliz, J. (19 de abril de 1959). La Universidad del Centro del Perú. La Voz de Huancayo, p. 9.

Zapata, A. y Garfias, M. (2014). Apuntes de una historia de organización y lucha 19692014. FNTMMP. URL: https://issuu.com/federacionminera/docs/librohistoria-fntmmsp-260914-para-web

\section{CITAR COMO:}

Lázaro Aquino, T. G. (2022). Origen comunal e institucionalización de la Universidad Nacional del Centro del Perú. Puriq, 4, e235. https://doi.org/10.37073/puriq.4.1.235 